\title{
Atmospheric Response to SST Anomalies. Part I: Background-State Dependence, Teleconnections, and Local Effects in Winter
}

\author{
STEPHEN I. THOMSON AND GEOFFREY K. VALLIS \\ University of Exeter, Exeter, United Kingdom
}

(Manuscript received 6 October 2017, in final form 22 June 2018)

\begin{abstract}
The atmospheric response to SST anomalies is notoriously difficult to simulate and may be sensitive to model details and biases, particularly in midlatitudes. Studies have suggested that the response is particularly sensitive to a model's background wind field and its variability. The dependence on such factors has meant that it is difficult to know what responses, if any, are robust, and whether the system itself is sensitive or whether models themselves are failing. Our goal in this work is to better understand the geographical and seasonal dependence of the atmospheric response to SST anomalies, with particular attention to the role of the background state. We examine the response of an idealized atmospheric model to SST anomalies using two slightly different configurations of continents and topography. These configurations give rise to different background wind fields and variability within the same season and therefore give a measure of how robust a response is to small changes in the background state. We find that many of the midlatitude SST anomalies considered do not produce responses that are common across our model configurations, confirming that this problem is very sensitive to the background state. Local responses in the tropics, however, are much more robust. Some of the basic-state dependence seen in midlatitudes appears to be related to the structure of both the model's modes of internal variability and the stationary wave field. In addition, midlatitude responses involving a significant amount of vertical temperature advection produce larger-scale responses, consistent with recent studies of atmospheric responses near strong western boundary currents.
\end{abstract}

\section{Introduction}

The interaction between the atmosphere and the ocean is a complex and multifaceted problem. In addition to its intrinsic interest, the interaction is important for the prosaic reason of seasonal weather prediction. That there may be some predictability in the system is suggested by studies showing a correlation between autumntime SSTs and the following winter's phase of the North Atlantic Oscillation (Czaja and Frankignoul 2002) and between springtime SSTs and the following summer's phase of the summer North Atlantic Oscillation (Dong et al. 2013). Given this, albeit unconfirmed, source of such predictability, it is important to understand how the atmosphere responds to SST anomalies in each of the seasons, why the response appears to differ between these seasons (with winter seemingly being more predictable), and which locations of SST anomalies give rise to the strongest response. This study and its companion study,

Corresponding author: Stephen I. Thomson, stephen.i.thomson@ gmail.com
Thomson and Vallis (2018, hereafter Part II), will look at the atmospheric response to SST anomalies in Northern Hemisphere winter and summer, respectively, and compare the responses in these two seasons.

Climate processes involving the atmospheric response to tropical SSTs [e.g., El Niño-Southern Oscillation (ENSO)] are often regarded as fairly well captured by models, although biases in the detailed characteristics of ENSO remain (Bellenger et al. 2014). The basic character of the local atmospheric response in the region surrounding a tropical SST anomaly is well explained by the simple models of Matsuno (1966) and Gill (1980). The basic response to a warm anomaly on the equator is two low pressure centers on either side of the equator, with low-level eastward winds along the equator to the west of the anomaly and westward winds to the east of the anomaly, with the two components being associated with equatorial Rossby and Kelvin waves, respectively. SST anomalies off the equator give rise to a weaker Kelvin wave component, leading to a predominance of the westward wind anomaly and a single low pressure center [for further details, see chapter 8 of Vallis (2017)]. 
A response in the midlatitudes to a warm tropical Pacific in Northern Hemisphere winter is also well known, with Bjerknes $(1966,1969)$ finding a deepening of the Aleutian low in response to a warm tropical Pacific. The impact of tropical Pacific SST anomalies on the atmosphere is also seen to extend into the Atlantic, with Rossby waves generated at the equator providing teleconnections to the midlatitudes (Hoskins and Karoly 1981; Scaife et al. 2017). A so-called stratospheric pathway has also been proposed for communicating changes in the tropics to the midlatitudes, particularly in connection with El Niño and stratospheric sudden warmings (SSWs), the combination of which can result in a negative state of the North Atlantic Oscillation (NAO; Domeisen et al. 2015).

By contrast, the atmospheric response to midlatitude SST anomalies has been found to be of relatively small amplitude and therefore easily hidden by natural atmospheric variability (e.g., Kushnir et al. 2002). The basic character of the atmospheric response to midlatitude surface heating is low-level convergence, with an associated cyclonic circulation, and an upper-level divergence, with an associated anticyclonic circulation. The low-level cyclone is found shifted downstream from the heating because of the significant role of cold-air advection (e.g., Fig. 2 of Hoskins and Karoly 1981). Such a response is often referred to as a "linear" response to surface heating because of the lack of eddy involvement. When eddies play a significant role, this linear response becomes more barotropic, with anomalous downwelling leading to anomalous surface divergence, and the low-level cyclone becomes replaced by a low-level anticyclone (e.g., section 3 of Kushnir et al. 2002).

A consequence of the generally small response to midlatitude SST anomalies is that models do not produce a robust or consistent response. Not only do baroclinic eddies reduce the signal-to-noise ratio, but there appears to be a dependence of the response on the mean jet position (e.g., Brayshaw et al. 2008; Saulière et al. 2012) so that jet-position biases in models lead to different responses. It has also been found that some responses project onto modes of internal variability (Peng and Robinson 2001), meaning that models with differing modes of internal variability could give different responses.

One way to better understand the dependence of atmospheric responses on the background wind field and its modes of variability is to compare responses in nearidentical models with slightly different jet positions and variability, and this is the approach we adopt. Any differences in the responses between the models can then be attributed to the background wind field rather than to the differences between particular months or differences in parameterization schemes and the like. We create near-identical models by running one idealized GCM with two different configurations of land and topography, without changing any of the other model parameters, and this leads to two similar, but not identical, background wind fields within the same model. We then look at the atmospheric responses to several SST anomaly patterns in each of the model configurations, comparing the response of each configuration to the same SST anomaly. Responses that do not depend on the details of the configuration can be said to be "robust."

We will focus on the free-atmosphere responses to SST anomalies (i.e., those outside the boundary layer). Such responses are likely to be longer lasting, and therefore more useful for seasonal prediction, and will also be more amenable to interpretation through techniques such as Rossby wave tracing.

Our study is split into two parts. This paper, which is the first part, looks at robust and nonrobust atmospheric responses to SST anomalies in the Northern Hemisphere winter season [December, January, and February (DJF)]. This focus on DJF is in line with most previous work on the atmospheric responses to SST anomalies. Part II looks at the same problem in the Northern Hemisphere summer season [June, July, and August (JJA)]. The comparison between our winter and summer results is made in this second paper.

The outline of the present paper is as follows. Section 2 describes the model and its two different configurations; section 3 compares the wind climatology and variability of the two configurations; section 4 outlines our design for anomaly experiments; section 5 discusses the responses to tropical anomalies; section 6 discusses the responses to midlatitude anomalies; section 7 compares the responses to tropical and midlatitude SST anomalies; and section 8 discusses the results and draws conclusions.

\section{Model setup}

We construct our models using Isca (Vallis et al. 2018), an open-source framework for the construction of atmospheric models. The model constructed here is of intermediate complexity, with realistic radiative transfer, but with simple convection, boundary layer, and surface schemes. The mixed layer ocean uses prescribed heat transport ( $Q$ fluxes) to keep the basic-state SSTs close to an annually repeating but seasonally varying SST climatology from AMIP (Taylor et al. 2000). Further details of the model setup can be found in the appendix. The two configurations of continents and 
Simple

(a)

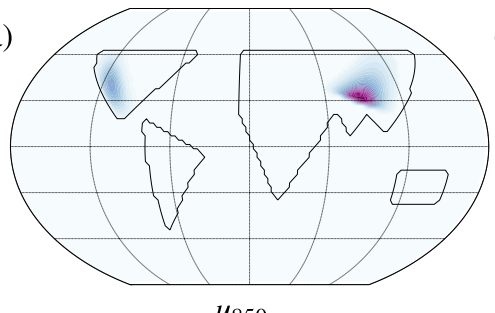

(d)



$u_{250}$

(g)



Complex

(b)

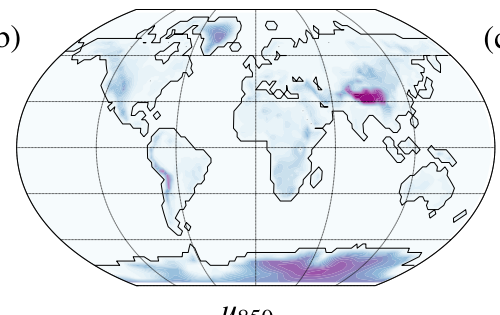

(c)

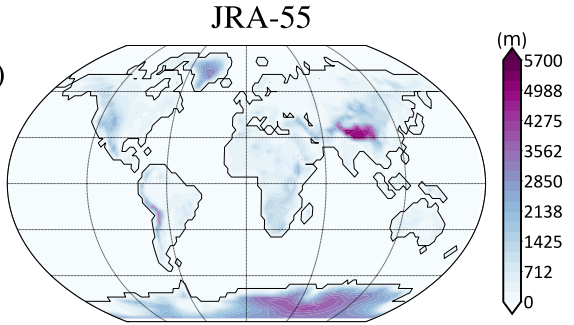

(e)

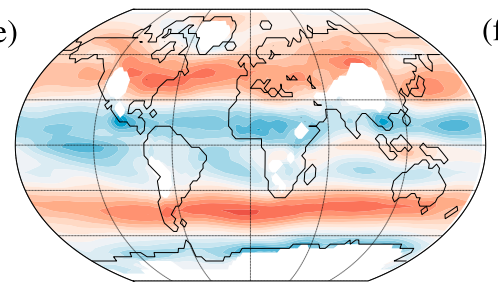

(f)

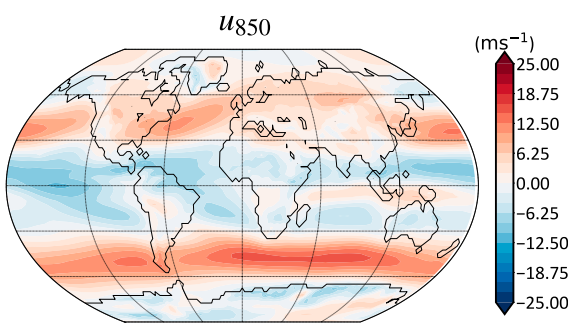

(h)

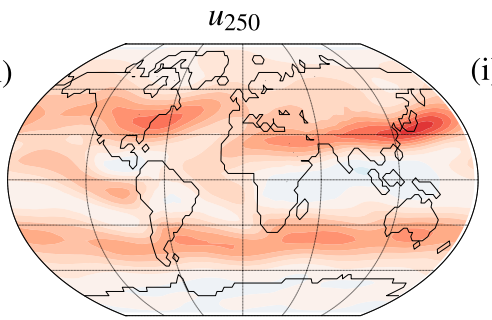

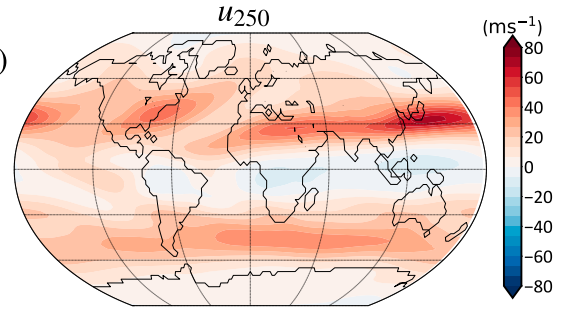

FIG. 1. A comparison of (a)-(c) the topographic height and the zonal wind at (d)-(f) 850 and (g)-(i) $250 \mathrm{hPa}$ in DJF in (left) the simple configuration, (center) the complex configuration, and (right) JRA-55. Model averages are taken over 20 years. JRA-55 averages are taken over the period 1958-2016.

topography are shown in Figs. 1a and $1 \mathrm{~b}$ and are referred to as the "simple" and "complex" configurations, respectively.

The different configurations of continents will give slightly different surface temperature gradients and therefore a slightly different spatial structure to, for example, the surface baroclinicity (not shown) and vertical temperature profiles (as in, e.g., Brayshaw et al. 2009). The different topographic distributions will affect how the jets flow over and around them, thereby affecting the stationary wave patterns. These features will help to differentiate the atmospheric states between the two configurations. A thorough investigation of the atmospheric responses to adding individual continents and mountain ranges in a realistic GCM can be found in Brayshaw et al. $(2009,2011)$.

In addition to the differentiating features described above, the complex configuration has a very simple representation of sea ice and land ice, whereas the simple configuration does not. We have taken the ice concentration dataset from the AMIP boundary condition dataset (Taylor et al. 2000) and have averaged over all years and all months to obtain an annual average distribution of ice. Using this data, the model's albedo is set to 0.7 in regions where the ice concentration is $>50 \%$, and the $Q$ flux is set to zero if the ice is over ocean. Other properties of the surface in these regions are determined by whether the underlying surface is land or ocean. The ice model further helps to distinguish the complex configuration's wind field from that in the simple configuration.

\section{Climatologies and variability of the model configurations}

\section{a. Climatologies}

We first establish that the two model configurations produce similar, but different, climatological jet properties and that these two configurations are both comparable to observed fields. Shown in Figs. 1d-f are the time-mean zonal winds at $850 \mathrm{hPa}$ in Northern Hemisphere winter (DJF) in the simple model configuration, the complex model configuration, and JRA-55 (Kobayashi et al. 2015), respectively. In terms of the broad wind structures, the two configurations of Isca and the reanalysis are remarkably similar in many aspects, although there are some notable differences. For example, the southwest-northeast tilt of the storm track at 
$850 \mathrm{hPa}$ is largest in JRA-55, less so in the complex configuration, and still less so in the simple configuration. Differences are also visible in the same fields at $250 \mathrm{hPa}$, shown in Figs. 1g-i. For example, the jet stream off the east coast of Japan is notably weaker and less zonal in the complex configuration compared with JRA-55 and the simple configuration. However, as at $850 \mathrm{hPa}$, the $250-\mathrm{hPa}$ climatologies display a broad similarity across the two configurations and JRA-55.

In terms of other features, the complex configuration has a noticeably weaker stationary wave pattern than does the simple configuration and JRA-55, particularly over the North Pacific, where the Aleutian low is particularly weak in the complex configuration. Further experimentation shows it is the addition of our ice model that causes the complex configuration's stationary wave pattern to be weak. A complex configuration run without the ice model in fact shows a stronger stationary wave pattern that is more similar to both the simple configuration and JRA-55. The weakening of the stationary wave pattern when the ice is introduced is due to the ice's higher albedo, which produces a colder global-mean surface temperature. The colder temperatures in turn produce tropical uppertroposphere cooling via the same lapse-rate effect that produces tropical upper-troposphere warming in climate change simulations (Vallis et al. 2015). With a cold tropical upper troposphere, the subtropical jet weakens, as does the midlatitude Eady growth rate, which results in a weaker eddy-driven jet and hence a weaker stationary wave pattern. The differences in the stationary wave patterns in the two configurations affect the configuration's stratospheres, with the Northern Hemisphere polar vortex in DJF being much stronger in the complex configuration than the simple configuration (not shown), consistent with the complex configuration's weaker stationary waves.

\section{b. Modes of variability}

To establish whether the models have different modes of internal variability, we calculate empirical orthogonal functions (EOFs) of monthly averaged atmospheric fields within each model configuration. All EOFs referred to shall be calculated using monthly averaged data and are referred to simply as "EOFs." The EOFs of, for example, zonal wind at $250 \mathrm{hPa}$ (not shown) are remarkably similar across the two configurations despite the continental and topographic differences.

In terms of the monthly variance explained by these EOFs, the model EOFs generally account for more of the variance than is explained in JRA-55. Over the Pacific, both configurations account for $\sim 10 \%$ more of the variance than in JRA-55, indicating that the model has fewer significant modes of internal variability compared with reanalysis, possibly indicating that it is more predictable. Over the
Atlantic, EOF1 in the simple configuration explains $\sim 15 \%$ more of the variance than JRA-55, while that in the complex configuration explains less. The latter is partly because $\mathrm{EOF} 2$ in the complex case is more similar to EOF1 in the simple case and JRA-55, indicating that the distribution of variance across modes is different in the complex configuration compared with the simple configuration and JRA-55.

In summary, the two model configurations both produce fields that bear comparison with reanalysis. They also produce fields that are similar to each other but not identical, thus allowing us to investigate the dependence of the response to an SST anomaly to relevant variations in the background state.

\section{c. Response to the Gulf Stream}

Whether or not a model is able to simulate the basicstate response to the Gulf Stream is thought to be an important indicator of whether it will be able to accurately simulate the forced response to SST anomalies (Minobe et al. 2008, 2010; Woollings et al. 2010). In Minobe et al. (2008), it was shown that a line of surface convergence over the warm edge of the Gulf Stream is apparent in observations, with the Laplacian of SLP bearing a strong relation to this convergence, unlike in models with a smoothed Gulf Stream temperature front, with the latter being a proxy for low-resolution models (see their Fig. 2c). The basic states in both our configurations, however, show a similar correlation between the Gulf Stream, surface convergence, and the Laplacian of sea level pressure (not shown), suggesting that the same mechanism is at work in our model as in the real world.

\section{Experiment design}

We first run each configuration to statistical equilibrium to give a "control" state, which we take as the state between the start of year 41 and the end of year 60 of a seasonally varying integration, with annually repeating forcing and boundary conditions. SST anomaly runs are spun off from the control state of the model at year 41 . We then create SST anomalies using an anomalous $Q$-flux field added to the seasonally varying $Q$-flux climatology in the mixed layer temperature equation [(A1)]. That is to say, the anomalies arise from an anomalous ocean heat transport, as in reality, and we maintain the significant advantages (and realism) of using a oceanic mixed layer rather than a specified SST distribution, as discussed by Bretherton and Battisti (2000).

Each anomalous $Q$ flux is constant in time and has the form

$$
\nabla \cdot Q^{\prime}=\left\{\begin{array}{cl}
A \gamma(\theta, \phi), & \gamma>0 \\
-\delta, & \text { elsewhere }
\end{array}\right.
$$


Here, $A=200 \mathrm{~W} \mathrm{~m}^{-2}$ is the amplitude of the anomaly, and $\gamma$ is the paraboloid function $\gamma(\theta, \phi)=1-\left[\left(\phi-\phi_{0}\right)^{2}+\right.$ $\left.\left(\theta-\theta_{0}\right)^{2}\right]$, where $\phi_{0}$ and $\theta_{0}$ are the central longitude and latitude of the anomaly, respectively. The constant $\delta$ is applied over the entire ocean outside of the paraboloid such that the area integral of $\nabla \cdot Q^{\prime}$ over the ocean is equal to zero. The SST outside the paraboloid region changes very little as a result of $\delta \neq 0$ (not shown). The amplitude of the $Q$-flux anomaly is constant in time, meaning the SST anomaly is present throughout the annual cycle. This allows us to look at winter responses and summer responses within the same annually repeating experiments.

The value $A=200 \mathrm{~W} \mathrm{~m}^{-2}$ has been chosen to give an SST anomaly of around $4^{\circ} \mathrm{C}$. This value is rather large compared to anomalies found in nature, particularly for those in the extratropics, but given the deliberately chosen small spatial extent of our SST anomalies (discussed below), this large value increases our signalto-noise ratio. Signal to noise is notoriously low in studies of the atmospheric response to extratropical SST anomalies, with internal variability typically being larger than the response to a typical anomaly (e.g., Kushnir et al. 2002), and a large-amplitude anomaly is needed if the spatial scale is small.

The spatial extent of the SST anomalies is determined by the values of $\phi_{0}$ and $\theta_{0}$, which we set for both to be $7.5^{\circ}$, meaning the anomalies are approximately six grid points in diameter at T42. We choose this small size in order to determine how SST anomalies in different parts of the ocean are able to produce a particular response, in essence like a coarse way of creating a Green's function for the model, as has been done successfully via a different method with a dry dynamical core model in Hassanzadeh and Kuang (2016). Our approach thus differs from previous studies that look at the response to specific large-scale SST anomalies [ENSO, Atlantic multidecadal oscillation (AMO), etc.]. Our aim is to determine how the atmosphere responds to small changes in the anomaly position, and we have therefore made the anomalies as small as we are reasonably able given our horizontal resolution. Our T42 horizontal resolution does not resolve these SST gradients especially well, which is of course a limitation (e.g., Parfitt et al. 2016), but the SST gradients associated with the anomalies are, in compensation, quite large. For each SST anomaly, we run the model for 24 years, discarding the first 4 years as a spinup phase. The remaining 20 years are then compared with 20 years from the control simulation for the relevant configuration, and time averages and other diagnostics are taken of the differences in various model fields. The statistical significance of any response is measured using the Student's $t$ test, with responses considered significant at the 95\% confidence limit.
Throughout this paper, we will be concerned with whether the responses to particular SST anomalies are robust. In order for a response to be deemed robust, we require that it meets two criteria:

1) The response in a particular quantity must be similar across the two configurations.

2) The responses within each configuration must be statistically significant, as judged by the $t$ test with a $95 \%$ confidence limit.

The responses produced by a particular SST anomaly in our two configurations can therefore have both robust and nonrobust components (i.e., the local responses in two cases might be the same across the configurations), but the remote responses may be different. We would then classify the local response as robust but the remote response as not being robust. In what follows, we will focus our analysis on the robustness of the zonal-wind responses at $250 \mathrm{hPa}$. We have chosen this level because of its usefulness for diagnosing the free-atmosphere responses, as these are more likely to be useful for teleconnections and predictability than shallow, boundary layer responses.

To gain an understanding of how the atmosphere responds to Atlantic and Pacific SST anomalies in the tropics and midlatitudes, we have chosen 31 different locations for our $Q$-flux anomalies, as shown in Fig. 2a. Each of the locations are run separately for each configuration, giving a total of 62 experiments. We label each experiment with a code (e.g., P5W). The first character in each label is either "P" or " $A$ ", denoting either the Pacific or Atlantic basin as the location for the anomaly. The second character is a number denoting the latitude, with the numbers 3,4 , and 5 representing latitudes $30^{\circ}, 40^{\circ}$, and $50^{\circ} \mathrm{N}$, respectively. The third character denotes the longitude of the anomaly. The midlatitude experiments are in groups of three per latitude per basin. If it is the central anomaly of the three, then the final character of the label will be "C." If it is the westernmost anomaly, then the final character is "W," and if it is the easternmost anomaly, then the final character is "E." In the tropical experiments, more anomalies are included per latitude per basin. In that case, an extra fourth character is introduced, being a number increasing from west to east.

Experiments P5W-P5E and A5W-A5E were placed at the entrance, middle, and exit regions of the controlclimate storm track in the Pacific and Atlantic, respectively, in order to test whether response will depend on the SST anomaly's location relative to the storm track itself (Peng and Whitaker 1999). Other anomaly locations were chosen to give the Pacific and Atlantic a reasonable coverage, including many equatorial locations in the Pacific. 

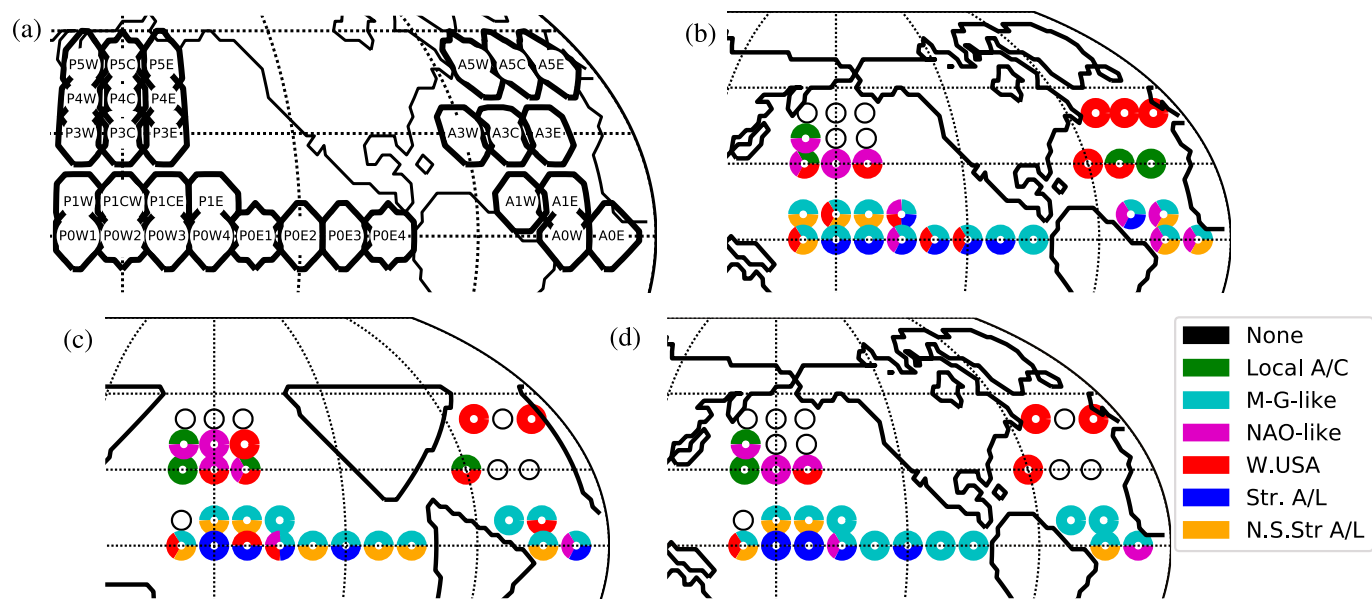

FIG. 2. (a) The name given to each location. Also shown is a summary of the various responses produced in each of the SST anomaly experiments. The variable used to define these responses is the DJF-mean zonal wind at $250 \mathrm{hPa}$. (b) The responses in each of the complex configuration experiments, with the colors denoting the type of response. (c) As in (b), but for the simple configuration. (d) The responses that are common across (b) and (c), which are the so-called robust responses.

\section{Responses to tropical anomalies}

To summarize the variety of responses, we have focused on the response of the zonal wind at $250 \mathrm{hPa}$ and have categorized the response in each case into as many of the following seven categories as are relevant:

- None of the below: The response displays none of the characteristics of the other six categories.

- Local anticyclonic circulation: A statistically significant anticyclone at $250 \mathrm{hPa}$, indicative of a local linear-like response as in, for example, Hoskins and Karoly (1981).

- Matsuno-Gill like: A statistically significant response displaying the broad characteristics of the simple linear responses to tropical heating described in Matsuno (1966) and Gill (1980), specifically, lowlevel eastward winds and upper-level westward winds.

- NAO like: A statistically significant response over the North Atlantic sector that looks like either a positive or negative NAO-like state. For some cases, this will constitute a local response, and in others (e.g., NAO-like responses to tropical Pacific SST anomalies), it will constitute a remote teleconnection.

- Anomaly off the western United States: A statistically significant small wind anomaly off the coast of Alaska.

- Strengthened Aleutian low: A statistically significant strengthening of the Aleutian low in the central North Pacific.

- Not-significant strengthened Aleutian low: A not statistically significant strengthening of the Aleutian low in the central North Pacific.
These categories represent the seven most common types of response across all the winter experiments. To represent the seven categories graphically, we have assigned each category a color and have color coded a circle in the SST anomaly's location by the kind of response they produce. Figure $2 \mathrm{~b}$ shows the responses produced by SST anomalies in the complex configuration. Figure $2 \mathrm{c}$ shows the responses produced by SST anomalies in the simple configuration. Figure $2 d$ shows the robust responses, being the responses to each SST anomaly that are common across both the configurations. The broad summary of this section is given by Fig. 2d, namely, that robust responses (at least locally) are found in all but one of the tropical SST anomaly cases. This is in stark contrast to the responses to the midlatitude anomalies, where around half of the anomalies did not produce a robust response.

\section{a. Extratropical responses to tropical Pacific SST anomalies}

Considering first the responses to SST anomalies in the tropical Pacific, one feature that ties 9 of the 12 cases together is that they produce a strengthening of the Aleutian low, consistent with Bjerknes (1966, 1969). An example of this is shown in Fig. 3, which shows the response of the zonal wind at $250 \mathrm{hPa}$ in case P0W4. The response in this case has other components in addition to the strengthened Aleutian low, and these are discussed further below. That such a standard result is reproduced in both our model configurations gives us confidence that both configurations are in a suitable regime for studying the atmospheric response to tropical SST anomalies. 

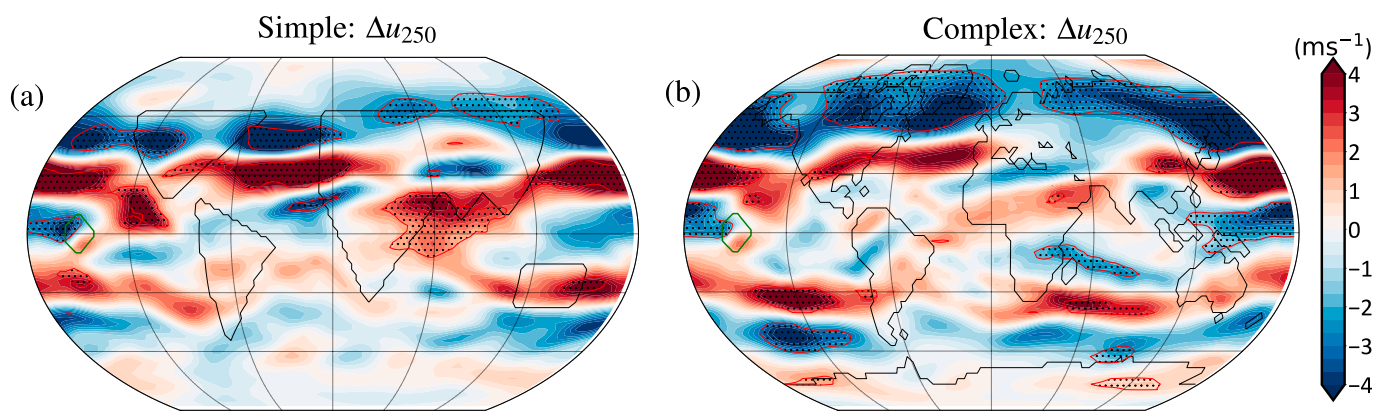

FIG. 3. Time-mean responses of the zonal wind at $250 \mathrm{hPa}$ to the $Q$-flux anomaly in case P0W4 in DJF for the (a) simple and (b) complex configurations.

\section{b. Tropical response to tropical Pacific SST anomalies}

We refer to the atmospheric responses in the tropical Pacific to SST anomalies in the tropical Pacific as "local" responses, given the proximity of the response to the SST anomaly itself. As can be seen in Fig. 2b, all of the tropical Pacific cases in the complex configuration produce a Matsuno-Gill-like response. The character of this response does vary with latitude, as predicted in the Matsuno-Gill model [as discussed in section 1; this can be seen graphically by comparing Figs. 8.14 and 8.11 of Vallis (2017)], with the off-equator cases producing only one low pressure center, rather than two on either side of the equator, and the lack of negative zonal winds at the surface to the east of the anomaly, as is associated with the Kelvin wave component of the response. Examples of a typical on-equator response and off-equator response at $250 \mathrm{hPa}$ in these cases are shown in Figs. 3 and 4 , respectively. The simple configuration has a similar prevalence of Matsuno-Gill-like responses throughout the tropical Pacific, with three exceptions: P1W, A1E, and P0W1. However, these three cases do show a weak Matsuno-Gill-like response in the simple configuration and so are not significant.

Leaving aside the well-understood contrast in responses between cases on and off the equator, it is clear from the above comparison that the overall character of the local responses to SST anomalies in the tropical Pacific does not change much with longitude or background state. However, the amplitude of the response can have some dependence on both longitude and background state. To see this, we note that the anomalous upwelling associated with the SST anomalies is much stronger above the anomalies in the west Pacific than the east Pacific, with the peak updraft velocities in cases P0W1, P0W2, and P0W3 being more than 3 times as large as those in cases P0E1, P0E2, and P0E3 in the complex configuration. A similar but slightly weaker contrast is seen in the same cases in the simple configuration. The transition in the magnitude of the updraft from strong to weak happens between cases P0W3 and P0W4 in both cases, consistent with the transition from midtroposphere upwelling to midtroposphere downwelling at around this longitude moving from west to east. This is consistent with previous work by Bony et al. (1997), as discussed in Lachlan-Cope and Connolley (2006), who suggest that updrafts in response to warm surface SSTs are strongest in areas of existing upwelling.

\section{c. Atlantic response to tropical Pacific SST anomalies}

One aspect of the atmospheric responses to tropical Pacific SST anomalies that is of particular interest is the projection of the response onto the NAO over the North Atlantic. One case that does this robustly is case P0W4, whose negative projection onto the NAO, as seen in Fig. 3, is consistent with the expected negative sign of the NAO projection from the warm Pacific during El Niño, as discussed in section 1.

Although case P0W4 does produce a robust jet shift over the North Atlantic, longitudinally adjacent cases P0W3 and P0E1 do not. The principal reason is that the simple configuration responds very differently in cases P0W3, P0E1, and P0W4, with the latter being the only case that produces a statistically significant jet shift over the North Atlantic. The complex configuration, by contrast, produces a consistent response over the North Atlantic in all three of these cases, but the jet shift is only statistically significant in case P0W4. The response of the northern Pacific across these three cases is quite consistent, unlike the response over the North Atlantic. This therefore suggests that the North Atlantic responses are much more sensitive to the exact details of the atmospheric state than are the responses in the Pacific. This is perhaps because of the background-state sensitivity of the propagation of the large-scale Rossby waves that are likely connecting the tropical Pacific and the North Atlantic in these cases. Linear Rossby wave tracing has 

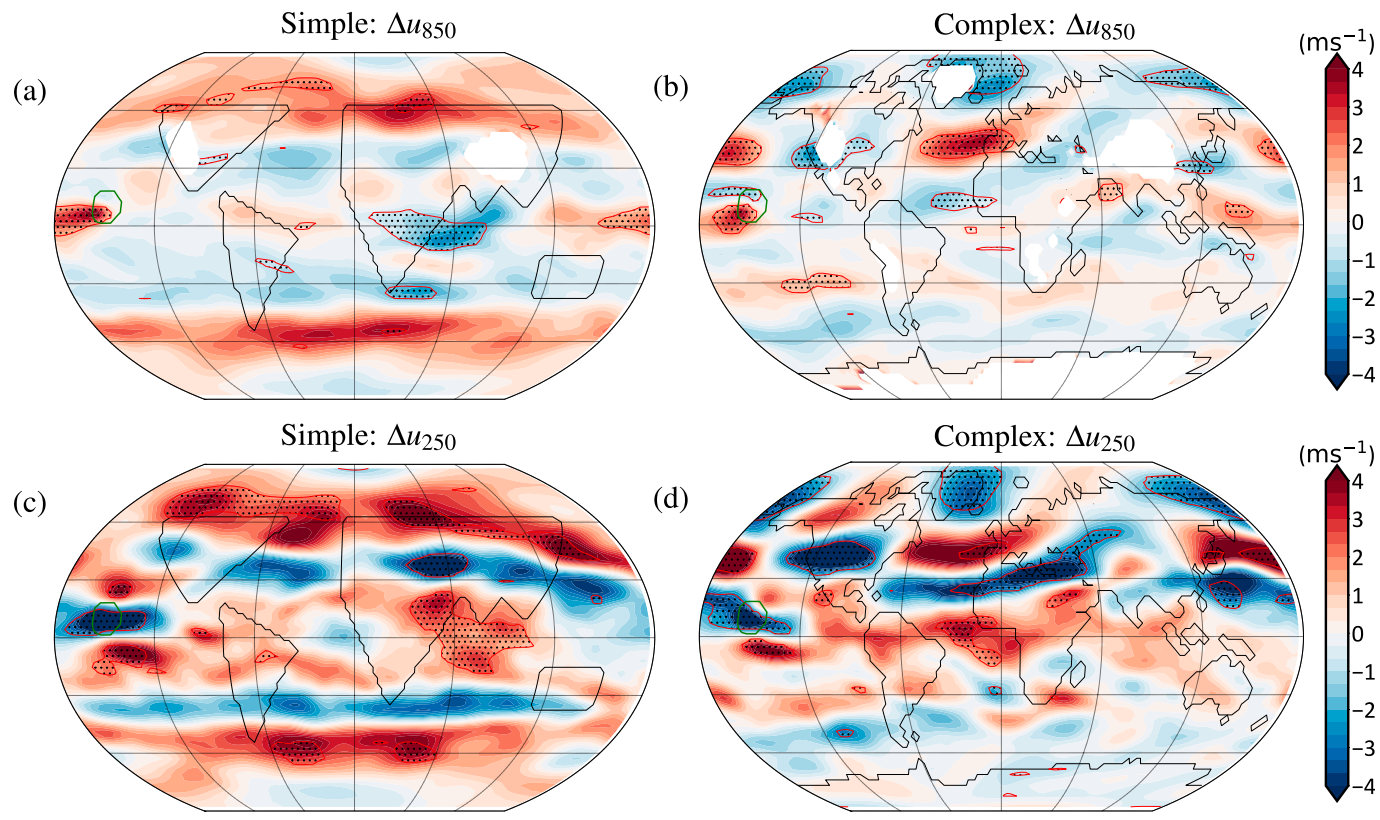

FIG. 4. Time-mean responses to $Q$-flux anomaly in case P1E in DJF for the (left) simple and (right) complex configurations at (a),(b) 850 and (c),(d) $250 \mathrm{hPa}$.

been performed, as in Hoskins and Karoly (1981), and confirms that this mechanism is a plausible explanation in this case but is not shown. The connection between the tropical Pacific and the midlatitude Pacific is clearly not as sensitive, judging by the ubiquitous strengthening of the Aleutian low described above.

\section{d. Responses to tropical Atlantic SST anomalies}

Local responses to SST anomalies in the tropical Atlantic are broadly similar to those in the Pacific, implying that on the most basic level, the local response to SST anomalies in the tropics is not much affected by whether it is in the Pacific or the Atlantic basin. However, finer details of the local responses, such as the longitudinal extension of the wind anomalies, and indeed their strengths do appear to vary with basin and with latitude and longitude.

In terms of remote connections, a negative NAO-like response is produced by all four tropical Atlantic cases in the complex configuration but only by one of the four in the simple configuration, again suggesting that tropicalmidlatitude teleconnections, possibly mediated by largescale Rossby waves, are highly sensitive to background wind conditions.

\section{e. Stratospheric response to tropical SST anomalies}

The model has a relatively realistic stratosphere, extending up to $0.03 \mathrm{hPa}$, so we briefly describe the stratospheric responses to our SST anomalies. All the cases in the complex configuration give a weakening of the vortex, apart from case P1CE, where the weakening is present but is not statistically significant. This comprehensive weakening is likely due to the increased strength of the Aleutian low in all the complex cases with the exception of case P1E. A strengthened Aleutian low is associated with stronger stationary waves in the troposphere, leading to increased upward planetary wave flux and thus disruption of the polar vortex, as in, for example, Hurwitz et al. (2012) and references therein.

In contrast, the simple configuration cases have a more varied set of stratospheric responses. A significant portion of the cases have a strengthened vortex, notably all those cases at $10^{\circ} \mathrm{N}$ in the Pacific (i.e., cases P1W-P1E) and all but case A0E from the four cases in the tropical Atlantic. Responses to anomalies along the equator in the Pacific vary between a wave- 1 oscillatory pattern on the polar night jet (five of the eight cases) to no response in cases P0W 2 and P0W3 and a strengthening in case P0E1. This sensitivity of the stratospheric response to longitude is in stark contrast to the insensitivity to longitude found in the stratospheric responses to equatorial Pacific SSTs in the complex configuration. However, the stationary wave field is (ironically) much more realistic in the simple configuration. We therefore interpret the responses in the simple configuration as being more realistic in stratospheric terms. There is, however, significant debate in the literature about what the realistic response of the stratosphere to tropical Pacific SST anomalies is, and how 


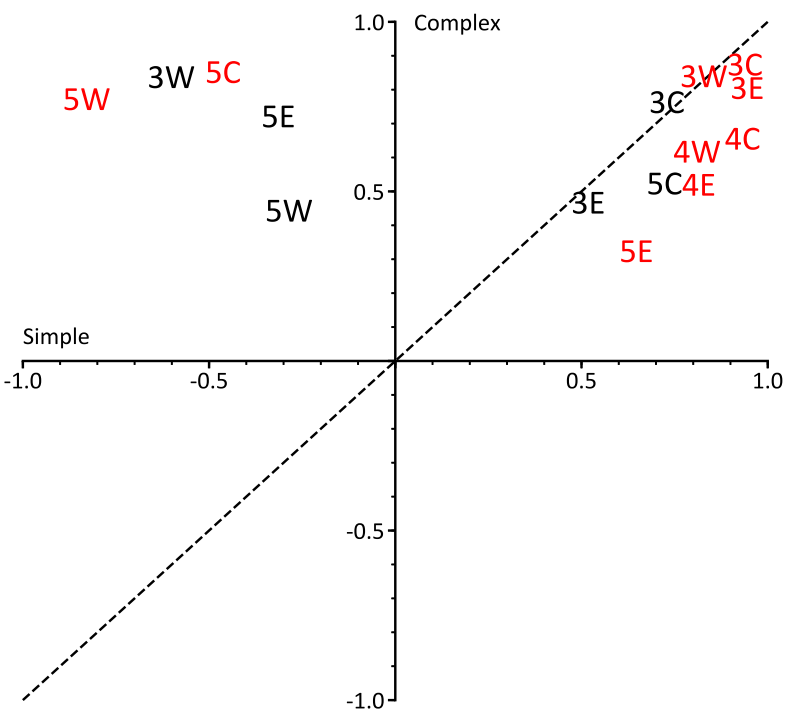

FIG. 5. Comparison of correlation coefficients in the simple configuration ( $x$ axis), with correlation coefficients in the complex configuration ( $y$ axis). The correlation coefficients are calculated between the zonal-wind response in DJF at $250 \mathrm{hPa}$ in cases with midlatitude SST anomalies and the EOF of zonal wind in DJF over the Atlantic basin $\left(80^{\circ} \mathrm{W}-40^{\circ} \mathrm{E}\right)$. The dashed line is $y=x$. The colors denote the basin in which the anomaly is placed, with red being in the Pacific and black being in the Atlantic. The codes used at each point correspond with those in Fig. 2 without the letter denoting the basin. It is noted that our classification of a robust response is based purely on the two criteria in section 4 and that this plot only serves as a guide as to how robust the projection onto the leading EOF is.

it depends on the longitudinal position of the SST anomaly, in regard to both the basic stratospheric state and stratospheric sudden warmings (e.g., Manzini et al. 2006; García-Herrera et al. 2006; Garfinkel et al. 2013; Iza and Calvo 2015; Li and Tian 2017). Further study of the model's diverse stratospheric responses is left to future work.

\section{Responses to midlatitude SST anomalies}

As described above, all but one of the tropical SST anomalies considered produce robust responses, at least locally, demonstrating the relative insensitivity of the responses to background climatology. By contrast, of all the midlatitude locations considered, only 7 out of the 15 midlatitude cases produce robust responses.

Of the eight cases that do not produce any robust responses, three are due to a configuration dependence (i.e., having a significant response in one configuration but not in the other), and five are because there was no statistically significant response in either configuration. This total lack of response at $250 \mathrm{hPa}$ in 5 of the 15 cases is consistent with previous work suggesting that the atmospheric response to midlatitude SST anomalies is often shallow and therefore confined to lower levels of the atmosphere [e.g., the prevalent shallow heating mode in Fig. 15b of Minobe et al. (2010)]. However, for all of the 15 midlatitude cases considered, no case produced a robust local response at $850 \mathrm{hPa}$ either, suggesting a general weakness of the low-level responses when compared with the high background variability found in and around the midlatitude storm tracks.

In terms of large-scale significant responses, there are several cases in each configuration that produce an NAO-like response over the North Atlantic, indicating a significant response within the free atmosphere. As a measure of the similarity of the response to the leading EOF in each basin, we calculate correlation coefficients between the zonal-wind response in the each of the 15 midlatitude cases at $250 \mathrm{hPa}$ and the EOF1 pattern of the zonal wind at $250 \mathrm{hPa}$ in the North Atlantic. Having calculated the coefficients in each case, and in each configuration, we plot the correlation coefficient in the simple configuration for each case against the correlation coefficient in the complex configuration for that same case, and this is shown in Fig. 5.

Those cases at $30^{\circ} \mathrm{N}$ in the Pacific (i.e., cases $\mathrm{P} 3 \mathrm{~W}$, $\mathrm{P} 3 \mathrm{C}$, and P3E, the latter of which is shown in Fig. 6) stand out for two reasons. First, the correlation between their responses and the EOF is high. And second, the correlation coefficients are high in both configurations, confirming our classification of these cases as giving robust responses according to the two criteria set out in section 4. Case P4W from the Pacific $40^{\circ} \mathrm{N}$ cases also stands out for these same reasons.

It is interesting to note, however, that all of the cases that produce an NAO-like response are forced in the Pacific rather than the Atlantic. This lack of Atlantic SST anomalies producing NAO-like responses is intriguing. It was suggested to the authors that this could indicate a lack of intrinsic Atlantic variability, such that the Atlantic is purely a slave to whatever is happening in the Pacific. We have subsequently checked the 2-6-day-filtered geopotential height variance in the Atlantic and Pacific sectors and compared them with the same diagnostic in JRA55 (not shown). This diagnostic indicates that our model configuration does have variability in the Atlantic reasonably comparable both to the Pacific and JRA-55, suggesting that there is some other explanation for the prevalence of NAO-like responses forced from the Pacific. This will be discussed further in section 7 .

In addition to those cases that have high and similar correlation coefficients in both configurations, it is interesting to note that some cases project strongly onto the EOF in one configuration but not in the other. For 



FIG. 6. As in Fig. 3, but for case P3E.

example, Fig. $5 \mathrm{c}$ shows that cases $\mathrm{P} 4 \mathrm{C}$ from the Pacific $40^{\circ} \mathrm{N}$ group and $\mathrm{A} 3 \mathrm{C}$ from the Atlantic $30^{\circ} \mathrm{N}$ group both project strongly onto the Pacific EOF in the simple configuration but hardly project at all in the complex configuration (not shown). These examples demonstrate that whether or not a response projects onto an EOF depends both on the location of the anomaly and the structure of the mode of variability itself, the latter of which is different between configurations.

\section{Stratospheric responses to midlatitude SST anomalies}

Although many of the midlatitude SST anomalies produce responses that are not robust, there are still interesting observations to be made. For example, cases P5W-P5E do not show significant tropospheric responses but do show statistically significant responses in the stratosphere. The response of the 10-hPa zonal wind in case P5C is shown in Fig. 7. The simple configuration shows a strengthening of the polar vortex, whereas the complex configuration shows a weakening of the polar vortex. Both of these results are statistically significant within each configuration but are not robust by our definition, as the two configurations respond with opposite signs. The changes in polar vortex strength are consistent with changes in the poleward eddy heat flux $\overline{\left(v^{\prime} T^{\prime}\right)}$ at $100 \mathrm{hPa}$, which is a measure of the wave driving of the stratosphere by upward-propagating planetary-scale waves. This flux has been shown to be highly correlated with stratospheric temperature and hence with polar vortex strength (Newman et al. 2001). In the simple case, a decrease in the eddy heat flux is seen in both December and January, with an increase in February. This 2-month decrease and 1-month increase is consistent with the winter-average vortex being stronger, as changes in heat flux have a delayed effect on the vortex strength (e.g., Fig. 1 of Newman et al. 2001). In the complex case, an increase in the heat flux is seen in DJF, consistent with the weaker vortex in this case.

The study of Hurwitz et al. (2012) used a chemistry climate model to show that positive SST anomalies in the North Pacific can weaken the Aleutian low and hence increase the strength of the polar vortex. Their result is therefore consistent with the response in our simple configuration but not that in the complex configuration. The Aleutian low in our simple configuration is much more realistic than the equivalent in our complex configuration (as discussed in section 3). This has the consequence that the upward-propagating flux of planetary waves into the stratosphere is more realistic in the simple configuration, making the polar vortex's strength more realistic. We believe this difference is responsible for the opposite response being found in the complex configuration. ${ }^{1}$ The opposite stratospheric responses in these cases underlines the importance of accurately representing the tropospheric stationary wave field when studying the stratospheric response to SST anomalies.

\section{Comparison of tropical and midlatitude responses}

The results described above show a clear contrast between responses to tropical SST anomalies and midlatitude SST anomalies in both complex and simple model configurations. That these responses are of different character is already well known, and there are several candidate explanations. We first consider the relative roles played by vertical and horizontal advection in response to surface heating.

\section{a. The role of vertical and horizontal advection}

The most common explanation for the differences between tropical and midlatitude responses to surface

\footnotetext{
${ }^{1} \mathrm{We}$ believe that these differences are likely not due to the different topographic distributions in the simple and complex configurations, as a complex configuration of Isca without the sea ice model gives a more reasonable stationary wave field and, by extension, a more reasonable stratospheric response, although the latter has not been tested.
} 

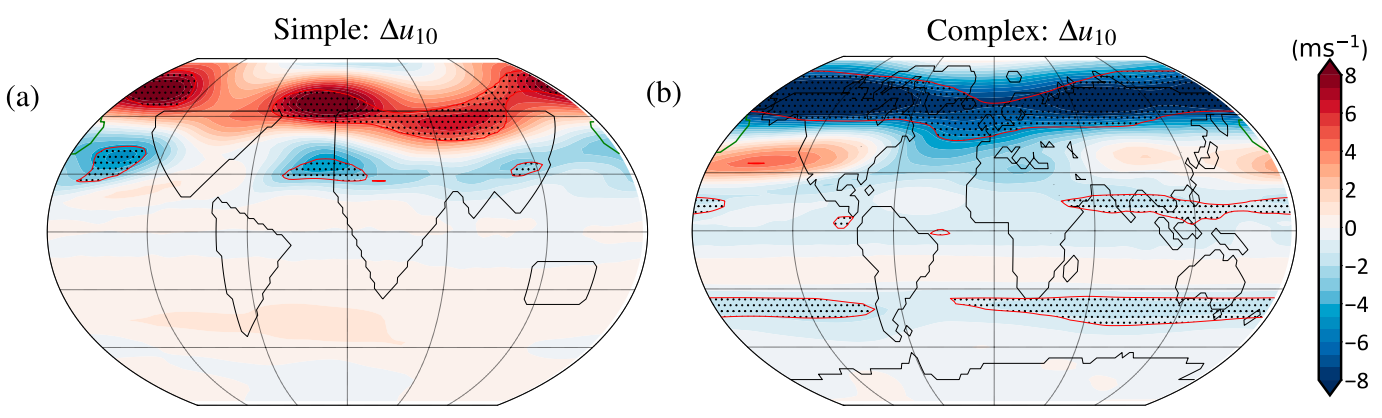

FIG. 7. As in Fig. 3, but at $10 \mathrm{hPa}$ in case P5C.

heating is that the response of the atmosphere to anomalous heating is predominantly vertical advection of anomalous temperature in the tropics and horizontal advection in the midlatitudes, as discussed in, for example, Hoskins and Karoly (1981) and Frankignoul (1985). The importance of horizontal advection in midlatitudes is because the significant equator-to-pole temperature gradient means that a southward flow over a warm SST anomaly will bring cold air down from higher latitudes to balance the heating and lead to a low pressure downstream of the heating [as seen in, e.g., Fig. 2 of Hoskins and Karoly (1981)].

Despite the importance of cold-air advection in midlatitude responses in Hoskins and Karoly (1981), it was noted in section 6 that the responses to our midlatitude SST anomalies were such that no surface low pressure centers were found at all. This is possibly because of the high low-level midlatitude variability. But one alternative possibility is that vertical advection is providing the dominant balance.

To investigate this contrast, we apply the linearized advection equation for temperature to our results, as in Eq. (39) of Frankignoul (1985):

$$
\frac{\bar{u}}{a \cos (\phi)} \frac{\partial T^{\prime}}{\partial \lambda}+\frac{v^{\prime}}{a} \frac{\partial \bar{T}}{\partial \phi}+\omega^{\prime} \frac{\partial \bar{T}}{\partial p}-\frac{R}{C_{p} p} \omega^{\prime} \bar{T}=\frac{q^{\prime}}{C_{p}} .
$$

Here, the primed quantities are departures from zonal means, $R$ is the gas constant, $C_{p}$ is the heat capacity at constant pressure, and $q$ is the combined diabatic heating and any effects from boundary layer frictional heating. We calculate explicitly all the terms on the lefthand side and find the right-hand-side terms as the sum of the terms on the left-hand side. Applying this decomposition directly to our anomaly experiments without subtracting the control fields captures the response to our SST anomalies but also captures the balances already in place in the climatology. We therefore calculate the terms in the anomaly experiments and the control experiments separately and look at differences in each of the terms between the anomaly and control experiments. It is these differences that we refer to as the responses of each term to our SST anomalies.

An example of the well-established tropical response is shown in Fig. 8a, showing the decomposition of these terms in the complex configuration's case P0W4. It is clear that the two terms involving $\omega^{\prime}$ are balancing the anomalous diabatic heating outside of the boundary layer, with little contribution from the horizontal advection terms.

By contrast, Fig. 8 b shows the same decomposition in the complex configuration's midlatitude case P3W. The profiles show some degree of similarity with the tropical case in the two $\omega^{\prime}$ terms, but the net contribution of these two $\omega^{\prime}$ terms is now of comparable strength to the meridional and zonal advection terms outside of the boundary layer, and as such, all the terms play a role in balancing the diabatic heating. However, near the surface, the cold-air advection argument of Hoskins and Karoly (1981) would suggest there should be a significant role for the horizontal advection terms. However, this is not seen, consistent with the lack of near-surface response noted above.

The work of Smirnov et al. (2015) uses the NCAR's CAM5 GCM to study the atmospheric responses to SST anomalies in the Oyashio extension region in the North Pacific and found that a version of their model with $1^{\circ}$ horizontal resolution produced a low-level circulation response very like that predicted by Hoskins and Karoly (1981), with a downstream surface cyclone, consistent with an important role for cold-air advection. However, in a simulation with $0.25^{\circ}$ horizontal resolution, there is a much smaller role for cold-air advection, with vertical advection becoming much more important (see their Fig. 11). Clearly, our model cannot claim to be resolving the atmospheric responses as well as either resolution of CAM5; however, it is plausible that our unusually strong SST anomalies are indeed strong enough to produce a response more like the high-resolution version of CAM5. 

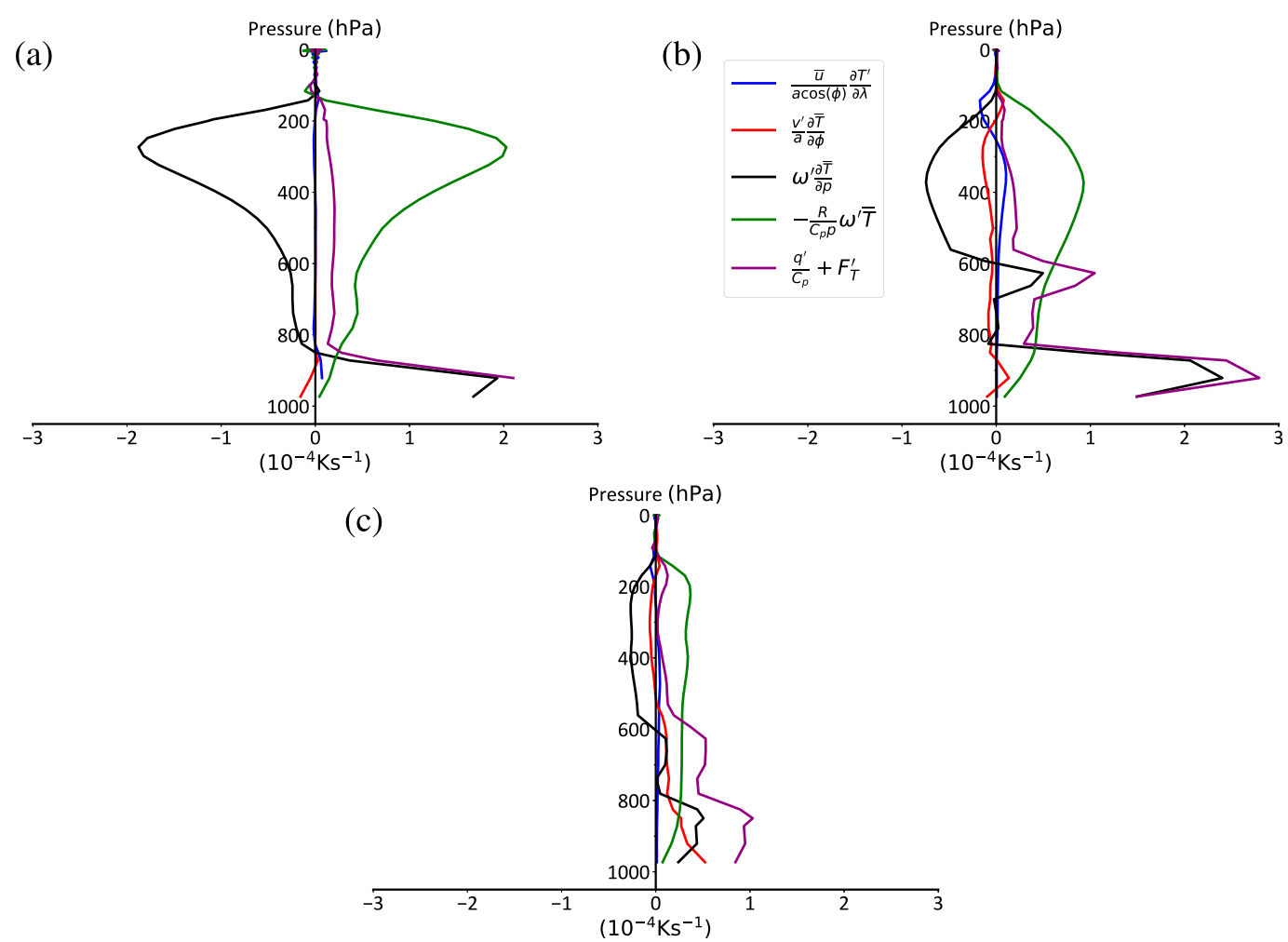

FIG. 8. (a),(b) A decomposition of Eq. (2), which is the linearized temperature advection equation, into its different terms; (a) shows the decomposition for a single horizontal grid point at the center of the SST anomaly in case P0W4 in the complex configuration. In contrast, (b) shows the same decomposition over the central grid point in case $\mathrm{P} 3 \mathrm{~W}$ in the complex configuration. (c) The same decomposition is shown for case $\mathrm{P} 3 \mathrm{~W}$ in the simple configuration.

It is also possible, however, that our model is getting this "realistic" midlatitude response for the wrong reasons. The reasons cited for Smirnov et al.'s (2015) qualitatively different behavior at high resolution is the improved representation of midlatitude storms and their associated transport of heat and moisture. This importance of short-time-scale fluctuations is also hinted at in Minobe et al.'s (2010) Fig. 17a, where it is shown that a significant amount of the temperature advection is taking place through fluctuations on a submonthly time scale. Our temperature advection analysis in Fig. 8 is done using monthly mean data, but use of daily mean data makes only a tiny quantitative difference to the results, suggesting that storms are not as heavily involved in this response as in the high-resolution model of Smirnov et al. (2015) or in reality. Further investigation is required.

Having identified a realistic vertical-advectiondominated response in midlatitudes, the sensitivity of this kind of response to background state can be tested by comparing the above to case $\mathrm{P} 3 \mathrm{~W}$ in the simple configuration. The decomposition in this case is shown in Fig. 8c. All of the terms in the budget are noticeably smaller in the simple configuration's case P3W apart from the meridional advection term, which is a significant contributor to the balance near the surface. The sign of this term suggests the presence of cold-air advection in this response. According to our categorizations in Fig. 2, it is seen that case P3W in the complex configuration produces anomalies over the western United States and the North Atlantic, where in the simple configuration case, $\mathrm{P} 3 \mathrm{~W}$ only produces a local anticyclone aloft. We therefore find that a significant role for vertical advection is seen alongside a global response, whereas cold-air advection is seen alongside a local response. This result is consistent with, for example, Fig. 15 of Smirnov et al. (2015), who show a basin-scale response in their high-resolution simulations of the Oyashio extension region, with its significant role for vertical advection, but only a local response in the low-resolution simulation with significant cold-air advection. A circumglobal response to midlatitude surface heating with significant vertical advection has also recently been shown in a 
lead-lag regression study with reanalysis data by Wills et al. (2016).

The large-scale, NAO-like response in case P3W in the complex configuration is the type of teleconnection that may be useful for seasonal prediction of the NAO. However, it is not enough for a response to be large scale for it to be useful for seasonal prediction. The response must also decay on a longer time scale than typical weather fluctuations, lasting for weeks or longer. It is not possible to directly measure the decay time of the responses in our experiments, as the SST anomalies are present throughout the year. This means that any response we see in a particular season could have been forced by the current SST anomaly, or last season's SST anomaly, or a combination of the responses over many time scales. We can, however, determine how long the response is present within the winter season by looking at the responses on a monthly time scale. In case P3W, a statistically significant movement of the jet stream over the North Atlantic is present in the complex configuration's monthly mean responses from October through April, with the pattern being broadly consistent across these months (not shown). In the simple configuration, however, no such North Atlantic response is seen in any of these months. This suggests that the response in the complex configuration is long lasting and may therefore be useful for seasonal prediction. Further experiments with time-varying SST anomalies are required to investigate this possibility.

\section{b. Storm tracks and the NAO}

It has been proposed that an extratropical SST anomaly will not produce a significant NAO-like response unless it perturbs the storm tracks (Peng et al. 2003). The idea behind this statement is that the NAO is driven primarily by transient eddy vorticity fluxes (e.g., Feldstein 2003; Vallis et al. 2004), and therefore, in order for an extratropical SST anomaly to produce a significant NAO-like response, the anomaly needs to perturb the transient eddy vorticity fluxes. Peng and Whitaker (1999) suggests that an SST anomaly does this by inducing an anomalous diabatic heating, which drives an anomalous flow, which then interacts with the storm track, resulting in perturbed eddy vorticity forcing, which then drives an NAO-like anomalous flow.

In all our midlatitude cases, no significant responses were seen in the transient eddy vorticity fluxes, including those where an NAO-like response was in fact observed (e.g., cases P3W, P3C, and P3E). Possible reasons for this lack of response include the small spatial scale of our SST anomalies, being much smaller than the SST anomalies used in Peng and Whitaker (1999) and Peng et al. (2003). The small spatial scales of our SST anomalies may give rise to only a minimal interaction with the storm track, implying that the responses observed in our experiments are largely due to the anomalous diabatic heating and its associated anomalous flow, not the effects of eddy fluxes.

The recent work of Brayshaw et al. (2011) and Saulière et al. (2012) find storm-track changes in response to the presence of large-scale SST anomalies, and they argue that these storm-track changes are due to changes made to the large-scale latitudinal SST gradients. The changed gradients then change the surface baroclinicity and therefore change storm growth within the storm track. It seems likely, therefore, that our SST anomalies are on a spatial scale that is too small to significantly affect these large-scale gradients, and therefore, an eddy-mediated response is not seen. Further exploration of this is a topic for future investigation but is beyond the scope of this paper.

As an additional attempt to understand the atmospheric responses to SST anomalies and how these are different between the tropics and the midlatitudes, we now consider the SST variability that is associated with the first EOF of the zonal wind over the North Atlantic, shown in Fig. 9. Peng et al. (2003) proposed that the shape of the NAO-associated SST tripole seen in these regressions is indicative of the regions of the ocean where the SST has the most influence on the atmosphere, and vice versa. If this hypothesis is correct, then Fig. 9a suggests that SST anomalies in the regions of these tripole maxima should be in prime locations for producing a significant atmospheric response in the simple configuration, and the same for Fig. $9 \mathrm{~b}$ for the complex configuration. However, of all the SST anomalies in the Atlantic basin that were considered in the present work, it is only those in the tropics that produce any robust statistically significant responses over the North Atlantic, and further than that, no single configuration produced an NAO-like response to a North Atlantic SST anomaly. One possible reason for our anomalies in these areas not producing robust responses is that the response to the SST tripole is nonlinear, meaning that the responses to each pole of the tripole individually do not add up to the response to the whole tripole. Therefore, it may be that the response to our warm SST anomalies in isolation will not produce as much of a response as would the sum of multiple anomalies. Further work is necessary to investigate this possibility.

\section{c. Latitude and longitude dependences in midlatitudes}

Some of our midlatitude SST anomalies evidently can produce responses that project onto the model's internal 

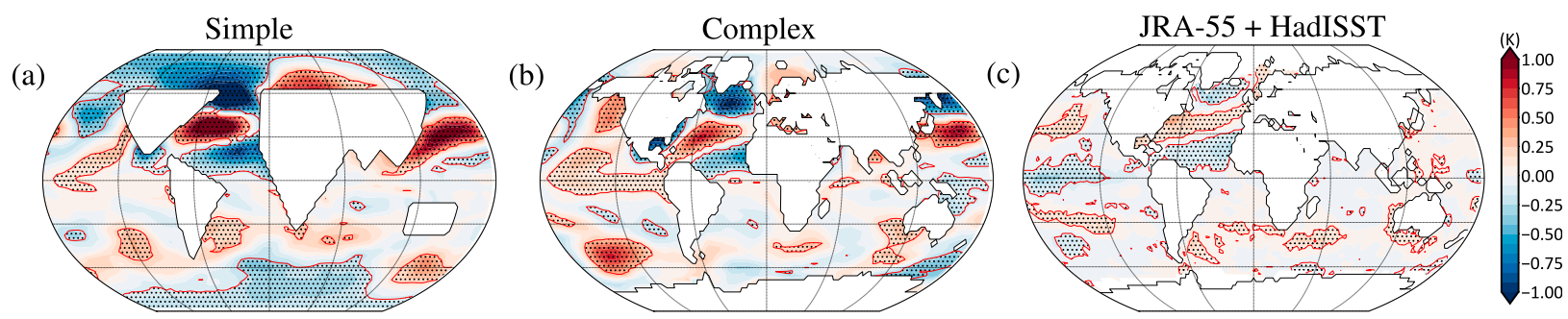

FIG. 9. (a) The linear regression coefficients of the simple configuration's SST regressed onto the simple configuration's PC1 time series of the first EOF of the zonal wind at $250 \mathrm{hPa}$ over the Atlantic basin. The statistical significance of the regression coefficients are calculated using the two-sided Student's $t$ test, and areas above the 95\% significance level are shown with stippling. (b) As in (a), but for the complex configuration. (c) As in (a), but for the HadISST dataset for the SSTs and the PC1 time series calculated from JRA-55 between 1958 and 2015 .

modes of variability. It is noteworthy from our midlatitude experiments, however, that whether the response projects onto such modes is sensitive to the latitude and longitude of the anomaly, as demonstrated by the range of correlation coefficients seen for the midlatitude cases in Fig. 5. As a complementary viewpoint to the transient eddy flux picture discussed above for the NAO, it has been proposed that whether or not a response projects onto an EOF may be related to the SST anomaly's location relative to the minima/maxima in the EOF pattern (Peng and Robinson 2001). The responses in our Pacific midlatitude cases partially support this conclusion. The hemisphere-wide EOF1 of the geopotential height has minima in both simple and complex configurations over the midlatitude Pacific, with the minimum in the simple configuration at $850 \mathrm{hPa}$ sitting at approximately $35^{\circ} \mathrm{N}, 177^{\circ} \mathrm{W}$, and the minimum in the complex configuration at $850 \mathrm{hPa}$ sitting at approximately $40^{\circ} \mathrm{N}, 177^{\circ} \mathrm{W}$. The fact that this minimum is farther to the north in the simple configuration would suggest that anomalies farther north should project more strongly onto the EOF in the simple configuration than in the complex configuration. This prediction does seem to be somewhat borne out in the results, with two cases at $40^{\circ} \mathrm{N}$ producing NAO-like responses in the simple configuration but only one case at $40^{\circ} \mathrm{N}$ in the complex configuration. However, there is also somewhat of a longitude dependence in the anomalies that do and do not produce NAO-like responses, suggesting that this EOF picture is not a complete explanation. Alternatively, we identify that differences in the responses are also consistent with differences in the stationary wave field in the two configurations. In contrast to the EOFs, the stationary wave field does show a significant longitude shift between configurations. The Pacific minimum in $850-\mathrm{hPa}$ geopotential height field including only waves $1-3$ has a minimum that is $10^{\circ}$ farther west in the complex case compared with the simple case (not shown). An example of a case where this might be important is $\mathrm{P} 4 \mathrm{C}$, where the response in the simple configuration projects strongly onto longitudinal wavenumbers 1 and 2 , where the response in the complex configuration does not. Based on this observation, we argue that proximity to the minima/ maxima in the stationary wave field could be as important for creating an EOF-like response as proximity to the EOF's own minima/maxima.

\section{Discussion and conclusions}

Our goal in this paper has been to better understand the response of the atmosphere to localized SST anomalies, with particular reference to the dependence of the responses on different background states in both the tropics and midlatitudes. Here, we have focused on winter, and in a companion paper, Part II, we look at summer and provide a comparison. We have used two slightly different configurations of the same idealized GCM and compared their responses to SST anomalies in many locations across the tropical and midlatitude Pacific and Atlantic. Our SST anomalies are deliberately small in geographic extent but large in amplitude, to obtain the cleanest possible response. Our measure of the robustness of a particular response is that a similar response is produced in both configurations and that the response is significant within each configuration. Given this definition of robustness, one of the main conclusions of this work is that all of the tropical anomalies considered do produce robust local responses in DJF, whereas the majority of midlatitude anomaly locations considered do not produce robust local responses. Furthermore, a number of cases in both the tropics and the midlatitudes also produce robust remote responses, although these are found to be highly sensitive to both location and background state.

The relative insensitivity to background state of the character of the local responses in the tropics is in contrast to a significant longitudinal contrast in the strength of the responses, with anomalous updraft velocities in 
the west Pacific being significantly higher than those in the east Pacific. As discussed in section 5b, this is consistent with previous work by Bony et al. (1997), who suggest that updrafts in response to warm surface SSTs are strongest in areas of existing upwelling, like the western Pacific. The fact that we also see significant anomalous updrafts in the east Pacific may be partly due to the large amplitude of our SST anomalies, which may be strong enough to trigger tropical deep convection in all locations. Further work is required to investigate this possibility.

In terms of remote responses to tropical anomalies, the strengthening of the Aleutian low in response to heating the tropical Pacific is remarkably robust. This is robust in the sense that it is insensitive to the SST anomaly location within one configurations but also insensitive to the background wind changes between the configurations. The responses in the simple cases are often weaker than the complex cases, however, and this may have something to do with the general weakness of the Aleutian low in the complex configuration, as discussed in section 3. This does, however, underline the importance of getting a model's basic state right if the responses of a model are to be both realistic and reproducible in other models.

In terms of the local responses to midlatitude anomalies, very few cases produced any local responses of significance, and even fewer of them were robust. This is particularly the case in locations with high background variability (e.g., within the midlatitude storm tracks). The responses of the most significance in the midlatitudes were those that projected onto the model's modes of internal variability and particularly the NAO over the North Atlantic. Insight from the temperature advection equation has shown in the example case of $\mathrm{P} 3 \mathrm{~W}$ that the complex configuration produced an NAOlike response, seemingly thanks to the dominance of the vertical advection term and the absence of horizontal cold-air advection. In the simple configuration, however, an NAO-like response was not produced, consistent with a much weaker vertical advection term and a more significant role played by low-level cold-air advection. This is consistent with ideas about the importance of the vertical advection term for producing large-scale responses (e.g., Wills et al. 2016; Smirnov et al. 2015; Minobe et al. 2010). How these conclusions hold up in a higher-resolution model, and the resultant backgroundstate dependence, is a topic for future work.

In addition to the temperature advection equation explanation, several other explanations present themselves for the production of NAO-like responses in some midlatitude cases rather than others. The first is simply that regions of low background variability are more likely to produce robust responses, and our results are certainly consistent with this idea. The second is that the spatial structure of the model's internal modes of variability differ between configurations, meaning a given SST anomaly could be closer to an antinode in one of the configurations, thus giving a larger response. The third is that the stationary wave patterns differ between configurations, again meaning a given SST anomaly could be closer to an antinode in one of the configurations, thus giving a larger response. The first of these ideas is well accepted, but both the second and third explanations are less widely accepted, and both are supported by different sets of our experiments. We have not been able to discern which of the second and third explanations is more widely applicable in the real world, and further work is required to investigate this.

One of the main conclusions from this comparison of configurations is that the stationary wave field is particularly important for getting the correct response to SST anomalies. This is true not only for the tropospheric component of the response as discussed above but also for those responses within the stratosphere, for which the long-wavelength stationary waves are particularly important.

Overall, our results confirm the paradigm, discussed in, for example, Kushnir et al. (2002), that responses to tropical anomalies give rise to more robust responses than do midlatitude anomalies. The reasons for this, in our experiments, are as follows:

- A tropical SST anomaly can reach the free atmosphere more easily than a midlatitude anomaly because of the significant role for vertical advection, as discussed in, for example, Hoskins and Karoly (1981) and highlighted in our experiments by the insensitivity of this result to our simple and complex configurations.

- The response to a tropical anomaly is less sensitive to changes in the background climatology than a midlatitude anomaly, for which there are many plausible explanations discussed above. This contrast is highlighted in our experiments by the consistency of tropical responses across our configurations but a significant lack of consistency in midlatitude responses across our configurations.

- Midlatitude SST anomalies exist in a highly variable environment with a low signal-to-noise ratio, as previously discussed in, for example, Kushnir et al. (2002), and highlighted in our experiments by the significant responses in the Pacific at $30^{\circ} \mathrm{N}$, where background variability is low.

For all these reasons (and the fact that real-world tropical SST anomalies are larger and longer lasting 
than their midlatitude counterparts), tropical SST anomalies provide much promise for seasonal predictability than midlatitude ones. However, our work also suggests that midlatitude responses occurring where the vertical advection term dominates are able to produce large-scale responses and so may also be useful for seasonal prediction. Evidently, understanding when and why the vertical term dominates is important for improving seasonal predictability, particularly with regard to the role played by horizontal resolution. Relating our work, with its relatively low horizontal resolution and spatially small but large-amplitude SST anomalies, to more realistic models with more realistic SST anomalies will help to better understand these effects.

Acknowledgments. We thank Mat Collins, Issac Held, Christopher O'Reilly, and Tim Woollings for helpful discussion. We also thank Martin Jucker for his help with the initial setup of the MiMA code and James Penn, Ruth Geen, Greg Colyer, and Penelope Maher for their continued collaboration on the Isca system. SIT is supported by the Natural Environment Research Council (Grant NE/M006123/1), and GKV acknowledges support from the Royal Society (Wolfson Foundation), the Leverhulme Trust, and the Newton Fund. Documentation and code required to run the Isca model framework can be found online (at www.exeter.ac.uk/isca and www. github.com/ExeClim/Isca).

\section{APPENDIX}

\section{Model Details}

Isca uses a spectral primitive equation dynamical core that we configure with 40 vertical levels extending up to $0.03 \mathrm{hPa}$ and at $\mathrm{T} 42$ horizontal resolution. Radiative transfer is calculated using the Rapid Radiative Transfer code RRTM (Clough et al. 2005). The radiative transfer uses a prescribed, zonally and hemispherically symmetric, annual-mean ozone distribution (as in Fig. 2 of Jucker and Gerber 2017). In its current configuration, Isca uses the Monin-Obukhov boundary layer scheme, the Betts-Miller convection scheme (Betts and Miller 1986), and a mixed layer ocean. The ocean has a seasonally varying horizontal heat transport, or " $Q$ flux," with a temperature equation given by

$$
c \frac{\partial T}{\partial t}=\mathrm{SW}+\mathrm{LW}-\mathrm{Sens}-\text { Latent }+\nabla \cdot Q,
$$

where $c$ is the mixed layer's heat capacity; $T$ is the mixed layer's temperature; $t$ is time; SW and LW are the net shortwave and longwave radiative fluxes, respectively;
TABLE A1. Table of model parameters.

\begin{tabular}{lcc}
\hline \multicolumn{1}{c}{ Parameter name } & Ocean & Land \\
\hline Albedo & 0.25 & 0.325 \\
Roughness length (m) & $2 \times 10^{-4}$ & $2 \times 10^{-3}$ \\
Mixed layer depth (m) & 20 & 2 \\
Evaporative resistance & 0.7 & 1.0 \\
\hline
\end{tabular}

Sens is the sensible heat flux; Latent is the latent heat flux; and $\nabla \cdot Q$ is the $Q$ flux, being a spatially and temporally varying two-dimensional vector that represents horizontal heat transport due to ocean dynamics. A model run with prescribed SSTs to calculate what $Q$ flux will be necessary to keep the free-running mixed layer SSTs close to those SST values, as in Russell et al. (1985). We take the prescribed SST values from the AMIP SST boundary conditions (Taylor et al. 2000), averaged to create a yearindependent, seasonally varying climatology.

In regions of land, we modify the surface to have a lower heat capacity, zero $\nabla \cdot Q$, a higher albedo, a larger surface roughness length, and an evaporative resistance, $\alpha=0.7$, where $\alpha$ appears in the standard Monin-Obukhov formula for surface evaporation flux $E$ as

$$
E=\rho_{a} C\left|v_{a}\right|\left(q_{a}-\alpha q_{s}^{*}\right) .
$$

Here, $\rho_{a}$ and $q_{a}$ are the atmospheric density and specific humidity in the lowest model layer, and $q_{s}^{*}$ is the saturation specific humidity calculated using the surface temperature [e.g., Eq. (11) in Frierson et al. (2006)]. Also, $C$ is the drag coefficient calculated from Monin-Obukhov similarity theory, and $\left|v_{a}\right|$ is the wind speed in the lowest model level. Having $0<\alpha<1$ over land inhibits evaporation, giving the effect of a limited water supply without the complications of a more realistic land surface model. Use of an alternative form for the evaporative resistance, $E=$ $\rho_{a} C\left|v_{a}\right| \alpha\left(q_{a}-q_{s}^{*}\right)$, was tried but made little difference to the climatologies. Parameters are given in Table A1.

The simple configuration of land and topography is based on the simplified continental outlines and Northern Hemisphere topography of Saulière et al. (2012). The complex configuration is simply the T42 ERA-Interim land mask and topography (Dee et al. 2011).

\section{REFERENCES}

Bellenger, H., E. Guilyardi, J. Leloup, M. Lengaigne, and J. Vialard, 2014: ENSO representation in climate models: From CMIP3 to CMIP5. Climate Dyn., 42, 1999-2018, https:// doi.org/10.1007/s00382-013-1783-z.

Betts, A. K., and M. J. Miller, 1986: A new convective adjustment scheme. Part II: Single column tests using GATE wave, BOMEX, ATEX and Arctic air-mass data sets. Quart. J. Roy. Meteor. Soc., 112, 693-709, https://doi.org/10.1002/qj.49711247308. 
Bjerknes, J., 1966: A possible response of the atmospheric Hadley circulation to equatorial anomalies of ocean temperature. Tellus, 18, 820-829, https://doi.org/10.3402/tellusa.v18i4.9712.

—, 1969: Atmospheric teleconnections from the equatorial Pacific. Mon. Wea. Rev., 97, 163-172, https://doi.org/10.1175/ 1520-0493(1969)097<0163:ATFTEP>2.3.CO;2.

Bony, S., K. M. Lau, and Y. C. Sud, 1997: Sea surface temperature and large-scale circulation influences on tropical greenhouse effect and cloud radiative forcing. J. Climate, 10, 2055-2077, https://doi.org/ 10.1175/1520-0442(1997)010<2055:SSTALS>2.0.CO;2.

Brayshaw, D. J., B. Hoskins, and M. Blackburn, 2008: The stormtrack response to idealized SST perturbations in an aquaplanet GCM. J. Atmos. Sci., 65, 2842-2860, https://doi.org/ 10.1175/2008JAS2657.1.

,-- , and ——, 2009: The basic ingredients of the North Atlantic storm track. Part I: Land-sea contrast and orography. J. Atmos. Sci., 66, 2539-2558, https://doi.org/10.1175/2009JAS3078.1.

,$- \ldots$, and $—$ 2011: The basic ingredients of the North Atlantic storm track. Part II: Sea surface temperatures. J. Atmos. Sci., 68, 1784-1805, https://doi.org/10.1175/2011JAS3674.1.

Bretherton, C. S., and D. S. Battisti, 2000: An interpretation of the results from atmospheric general circulation models forced by the time history of the observed sea surface temperature distribution. Geophys. Res. Lett., 27, 767-770, https://doi.org/ 10.1029/1999GL010910.

Clough, S., M. Shephard, E. Mlawer, J. Delamere, M. Iacono, K. Cady-Pereira, S. Boukabara, and P. Brown, 2005: Atmospheric radiative transfer modeling: A summary of the AER codes. J. Quant. Spectrosc. Radiat. Transf., 91, 233-244, https:// doi.org/10.1016/j.jqsrt.2004.05.058.

Czaja, A., and C. Frankignoul, 2002: Observed impact of Atlantic SST anomalies on the North Atlantic oscillation. J. Climate, 15, 606-623, https://doi.org/10.1175/1520-0442(2002)015<0606: OIOASA $>2.0 . \mathrm{CO} ; 2$.

Dee, D. P., and Coauthors, 2011: The ERA-Interim reanalysis: Configuration and performance of the data assimilation system. Quart. J. Roy. Meteor. Soc., 137, 553-597, https://doi.org/10.1002/qj.828.

Domeisen, D. I. V., A. H. Butler, K. Fröhlich, M. Bittner, W. A. Müller, and J. Baehr, 2015: Seasonal predictability over Europe arising from El Niño and stratospheric variability in the MPI-ESM seasonal prediction system. J. Climate, 28, 256271, https://doi.org/10.1175/JCLI-D-14-00207.1.

Dong, B., R. T. Sutton, T. Woollings, and K. Hodges, 2013: Variability of the North Atlantic summer storm track: Mechanisms and impacts on European climate. Environ. Res. Lett., 8, 034037, https://doi.org/10.1088/1748-9326/8/3/034037.

Feldstein, S. B., 2003: The dynamics of NAO teleconnection pattern growth and decay. Quart. J. Roy. Meteor. Soc., 129, $901-$ 924, https://doi.org/10.1256/qj.02.76.

Frankignoul, C., 1985: Sea surface temperature anomalies, planetary waves, and air-sea feedback in the middle latitudes. Rev. Geophys., 23, 357-390, https://doi.org/10.1029/RG023i004p00357.

Frierson, D. M. W., I. M. Held, and P. Zurita-Gotor, 2006: A grayradiation aquaplanet moist GCM. Part I: Static stability and eddy scale. J. Atmos. Sci., 63, 2548-2566, https://doi.org/10.1175/ JAS3753.1.

García-Herrera, R., N. Calvo, R. R. Garcia, and M. A. Giorgetta, 2006: Propagation of ENSO temperature signals into the middle atmosphere: A comparison of two general circulation models and ERA-40 reanalysis data. J. Geophys. Res., 111, D06101, https://doi.org/10.1029/2005JD006061.

Garfinkel, C. I., L. D. Oman, E. A. Barnes, D. W. Waugh, M. H. Hurwitz, and A. M. Molod, 2013: Connections between the spring breakup of the Southern Hemisphere polar vortex, stationary waves, and air-sea roughness. J. Atmos. Sci., 70, 21372151, https://doi.org/10.1175/JAS-D-12-0242.1.

Gill, A. E., 1980: Some simple solutions for heat-induced tropical circulation. Quart. J. Roy. Meteor. Soc., 106, 447-462, https:// doi.org/10.1002/qj.49710644905.

Hassanzadeh, P., and Z. Kuang, 2016: The linear response function of an idealized atmosphere. Part I: Construction using Green's functions and applications. J. Atmos. Sci., 73, 3423-3439, https://doi.org/10.1175/JAS-D-15-0338.1.

Hoskins, B. J., and D. J. Karoly, 1981: The steady linear response of a spherical atmosphere to thermal and orographic forcing. J. Atmos. Sci., 38, 1179-1196, https://doi.org/10.1175/15200469(1981)038<1179:TSLROA > 2.0.CO;2.

Hurwitz, M. M., P. A. Newman, and C. I. Garfinkel, 2012: On the influence of North Pacific sea surface temperature on the Arctic winter climate. J. Geophys. Res., 117, D19110, https:// doi.org/10.1029/2012JD017819.

Iza, M., and N. Calvo, 2015: Role of stratospheric sudden warmings on the response to central Pacific El Niño. Geophys. Res. Lett., 42, 2482-2489, https://doi.org/10.1002/2014GL062935.

Jucker, M., and E. P. Gerber, 2017: Untangling the annual cycle of the tropical tropopause layer with an idealized moist model. J. Climate, 30, 7339-7358, https://doi.org/10.1175/JCLI-D-17-0127.1.

Kobayashi, S., and Coauthors, 2015: The JRA-55 Reanalysis: General specifications and basic characteristics. J. Meteor. Soc. Japan, 93, 5-48, https://doi.org/10.2151/jmsj.2015-001.

Kushnir, Y., W. A. Robinson, I. Bladé, N. M. J. Hall, S. Peng, and R. Sutton, 2002: Atmospheric GCM response to extratropical SST anomalies: Synthesis and evaluation. J. Climate, 15, 2233-2256, https://doi.org/10.1175/1520-0442(2002)015<2233: AGRTES $>2.0 . \mathrm{CO} ; 2$.

Lachlan-Cope, T., and W. Connolley, 2006: Teleconnections between the tropical Pacific and the Amundsen-Bellinghausens Sea: Role of the El Niño/Southern Oscillation. J. Geophys. Res., 111, D2310, https://doi.org/10.1029/2005JD006386.

Li, Y., and W. Tian, 2017: Different impact of central Pacific and eastern Pacific El Niño duration of sudden stratospheric warming. Adv. Atmos. Sci., 34, 771-782, https://doi.org/10.1007/ s00376-017-6286-0.

Manzini, E., M. A. Giorgetta, M. Esch, L. Kornblueh, and E. Roeckner, 2006: The influence of sea surface temperatures on the northern winter stratosphere: Ensemble simulations with the MAECHAM5 model. J. Climate, 19, 3863-3881, https://doi.org/10.1175/JCLI3826.1.

Matsuno, T., 1966: Quasi-geostrophic motions in the equatorial area. J. Meteor. Soc. Japan, 44, 25-43, https://doi.org/10.2151/ jmsj1965.44.1_25.

Minobe, S., A. Kuwano-Yoshida, N. Komori, S.-P. Xie, and R. J. Small, 2008: Influence of the Gulf Stream on the troposphere. Nature, 452, 206-209, https://doi.org/10.1038/nature06690.

_- M. Miyashita, A. Kuwano-Yoshida, H. Tokinaga, and S. P. Xie, 2010: Atmospheric response to the Gulf Stream: Seasonal variations. J. Climate, 23, 3699-3719, https://doi.org/10.1175/ 2010JCLI3359.1.

Newman, P. A., E. R. Nash, and J. E. Rosenfield, 2001: What controls the temperature of the Arctic stratosphere during the spring? J. Geophys. Res., 106, 19 999-20 010, https://doi.org/ 10.1029/2000JD000061.

Parfitt, R., A. Czaja, S. Minobe, and A. Kuwano-Yoshida, 2016: The atmospheric frontal response to SST perturbations in the Gulf Stream region. Geophys. Res. Lett., 43, 2299-2306, https://doi.org/10.1002/2016GL067723. 
Peng, S., and J. S. Whitaker, 1999: Mechanisms determining the atmospheric response to midlatitude SST anomalies. J. Climate, 12, 1393-1408, https://doi.org/10.1175/1520-0442(1999)012<1393: MDTART $>2.0 . \mathrm{CO} ; 2$

— internal variability and the responses to an extratropical SST anomaly. J. Climate, 14, 2943-2959, https://doi.org/10.1175/ 1520-0442(2001)014<2943:RBAIVA > 2.0.CO;2.

,-- , and S. Li, 2003: Mechanisms for the NAO responses to the North Atlantic SST tripole. J. Climate, 16, 1987-2004, https:// doi.org/10.1175/1520-0442(2003)016<1987:MFTNRT>2.0.CO;2.

Russell, G. L., J. R. Miller, and L.-C. Tsang, 1985: Seasonal oceanic heat transports computed from an atmospheric model. Dyn. Atmos. Oceans, 9, 253-271, https://doi.org/10.1016/0377-0265(85)90022-3.

Saulière, J., D. J. Brayshaw, B. Hoskins, and M. Blackburn, 2012: Further investigation of the impact of idealized continents and SST distributions on the Northern Hemisphere storm tracks. J. Atmos. Sci., 69, 840-856, https://doi.org/10.1175/JAS-D-11-0113.1.

Scaife, A. A., and Coauthors, 2017: Tropical rainfall, Rossby waves and regional winter climate predictions. Quart. J. Roy. Meteor. Soc., 143, 1-11, https://doi.org/10.1002/qj.2910.

Smirnov, D., M. Newman, M. A. Alexander, Y. O. Kwon, and C. Frankignoul, 2015: Investigating the local atmospheric response to a realistic shift in the Oyashio sea surface temperature front. J. Climate, 28, 1126-1147, https://doi.org/10.1175/ JCLI-D-14-00285.1.

Taylor, K. E., D. Williamson, and F. Zwiers, 2000: The sea surface temperature and sea-ice concentration boundary conditions for AMIP II simulations. PCMDI Rep. 60, 25 pp.
Thomson, S. I., and G. K. Vallis, 2018: Atmospheric response to SST anomalies. Part II: Background-state dependence, teleconnections, and local effects in summer. J. Atmos. Sci., $\mathbf{7 5}$, 4125-4138, https://doi.org/10.1175/JAS-D-17-0298.1.

Vallis, G. K., 2017: Atmospheric and Oceanic Fluid Dynamics. 2nd ed. Cambridge University Press, 964 pp., https://doi.org/ $10.1017 / 9781107588417$.

_ - E. P. Gerber, P. J. Kushner, and B. A. Cash, 2004: A mechanism and simple dynamical model of the North Atlantic Oscillation and annular modes. J. Atmos. Sci., 61, 264-280, https://doi.org/10.1175/1520-0469(2004)061<0264: AMASDM $>2.0 . \mathrm{CO} ; 2$.

— , P. Zurita-Gotor, C. Cairns, and J. Kidston, 2015: Response of the large-scale structure of the atmosphere to global warming. Quart. J. Roy. Meteor. Soc., 141, 1479-1501, https://doi.org/ 10.1002/qj.2456.

—_ and Coauthors, 2018: Isca, v1.0: A framework for the global modelling of the atmospheres of Earth and other planets at varying levels of complexity. Geosci. Model Dev., 11, 843-859, https://doi.org/10.5194/gmd-11-843-2018.

Wills, S. M., D. W. J. Thompson, and L. M. Ciasto, 2016: On the observed relationships between variability in Gulf Stream sea surface temperatures and the atmospheric circulation over the North Atlantic. J. Climate, 29, 3719-3730, https://doi.org/ 10.1175/JCLI-D-15-0820.1.

Woollings, T., A. Charlton-Perez, S. Ineson, A. G. Marshall, and G. Masato, 2010: Associations between stratospheric variability and tropospheric blocking. J. Geophys. Res., 115, D06108, https://doi.org/10.1029/2009JD012742. 